\title{
Relationship between Personality and Insomnia in Panic Disorder Patients
}

\author{
Hae-Ran $\mathrm{Na}^{1}$, Eun-Ho Kang${ }^{1}$, Bum-Hee $\mathrm{Yu}^{1} \bowtie$, Jong-Min $\mathrm{Woo}^{2}$, Youl-Ri Kim², \\ Seung-Hwan Lee ${ }^{3}$, Eui-Jung Kim ${ }^{4}$, Sang-Yeol Lee ${ }^{5}$ and Sang-Keun Chung ${ }^{5}$ \\ ${ }^{1}$ Department of Psychiatry, Samsung Medical Center, Sungkyunkwan University School of Medicine, Seoul, Korea \\ ${ }^{2}$ Department of Psychiatry, Seoul Paik Hospital, Inje University School of Medicine, Seoul, Korea \\ ${ }^{3}$ Department of Psychiatry, Ilsan Paik Hospital, Inje University School of Medicine, Goyang, Korea \\ ${ }^{4}$ Department of Psychiatry, College of Medicine, Ewha Women's University, Seoul, Korea \\ ${ }^{5}$ Department of Psychiatry, Wonkwang University School of Medicine, Iksan, Korea
}

Objective Panic disorder (PD) is frequently comorbid with insomnia, which could exacerbate panic symptoms and contribute to PD relapse. Research has suggested that characteristics are implicated in both PD and insomnia. However, there are no reports examining whether temperament and character affect insomnia in PD. Thus, we examined the relationship between insomnia and personality characteristics in PD patients.

Methods Participants were 101 patients, recruited from 6 university hospitals in Korea, who met the DSM-IV-TR criteria for PD. We assessed sleep outcomes using the sleep items of 17-item Hamilton Depression Rating Scale (HAMD-17)(item 4=onset latency, item 5= middle awakening, and item $6=$ early awakening) and used the Cloninger's Temperament and Character Inventory-Revised-Short to assess personality characteristics. To examine the relationship between personality and insomnia, we used analysis of variance with age, sex, and severity of depression (total HAMD scores minus sum of the three sleep items) as the covariates.

Results There were no statistical differences $(\mathrm{p}>0.1)$ in demographic and clinical data between patients with and without insomnia. Initial insomnia (delayed sleep onset) correlated to a high score on the temperamental dimension of novelty seeking $3(\mathrm{NS} 3)\left(\mathrm{F}_{1,96}=6.93\right.$, $\mathrm{p}=0.03)$. There were no statistical differences $(\mathrm{p}>0.1)$ in NS3 between patients with and without middle or terminal insomnia.

Conclusion The present study suggests that higher NS3 is related to the development of initial insomnia in PD and that temperament and character should be considered when assessing sleep problems in PD patients.

Psychiatry Investig 2011;8:102-106

Key Words Personality, Temperament, Character, Insomnia, Panic disorder

\section{INTRODUCTION}

Panic disorder (PD), one of the quite common anxiety disorders, is often a disabling, chronic illness. ${ }^{1,2} \mathrm{PD}$ is frequently comorbid with insomnia, which could exacerbate panic symptoms and contribute to relapse of $\mathrm{PD}^{3}$ The association between insomnia and anxiety disorders, such as general anxiety disorder, post-traumatic syndrome disorder and $\mathrm{PD},{ }^{4-6}$ has been showed in many researches. Several researches showed

Received: September 20, 2010 Revised: February 11, 2011

Accepted: March 8, 2011 Available online: April 8, 2011

$\triangle$ Correspondence: Bum-Hee Yu, MD, PhD

Department of Psychiatry, Samsung Medical Center, Sungkyunkwan University School of Medicine, 50 Irwon-dong, Gangnam-gu, Seoul 135-710, Korea Tel: +82-2-3410-3583, Fax: +82-2-3410-6957, E-mail: bhyu@skku.ac.kr

(c) This is an Open Access article distributed under the terms of the Creative Commons Attribution Non-Commercial License (http://creativecommons.org/licenses/by$\mathrm{nc} / 3.0$ ) which permits unrestricted non-commercial use, distribution, and reproduction in any medium, provided the original work is properly cited. that insomnia is more commonly reported in those with PD than in normal individuals, ${ }^{7}$ and impaired sleep initiation and maintenance has been confirmed by polysomnographic studies. ${ }^{8}$ In addition, recurrent sleep panic attacks can create a state of anticipatory anxiety and apprehension over the prospect of yet another night of sleeplessness followed by another day of fatigue. ${ }^{9,10}$ The prevalence of insomnia in PD patients has been reported about 68 percent up to 93 percent ${ }^{6}$ possibly because of anxiety, depression ${ }^{11}$ and nocturnal panic attack. ${ }^{6}$

$\mathrm{PD}$ is frequently accompanied by agoraphobia and panic patients with agoraphobia are known to have a poorer prognosis, compared with those without agoraphobia. There had been few reports about the difference in the prevalence of insomnia between PD with agoraphobia and PD without agoraphobia. ${ }^{12,13}$

Insomnia can be caused by biological, psychological, and environmental predisposing factors and is known to also be affected by personality traits. ${ }^{14}$ However, the exact mechanism 
of insomnia in $\mathrm{PD}$ is still unknown. Researches have shown that both temperament and character are implicated in $\mathrm{PD},{ }^{15}$ and some studies also suggest a close relationship between certain, specific personality traits and insomnia. ${ }^{15-18}$ In addition to, several studies showed that personality disorders were implicated in PD. ${ }^{19-21}$ Personality disorders are common in subjects with PD and personality disorders have been shown to affect the course of PD. ${ }^{22}$ The prevalence and the effect on insomnia of personality disorders in PD patients are not well known till now.

Cloninger's personality theory ${ }^{14}$ provides an integrated concept for explaining psychopathology of PD, as well as that of insomnia. This model conceptualized personality as having 4 temperament dimensions [novelty seeking (NS), harm avoidance $(\mathrm{HA})$, reward dependence $(\mathrm{RD})$ and persistence $(\mathrm{P})$ ] and three character dimensions [self-directedness (SD), cooperativeness (C), and self-transcendence (ST)]. HA is known to play a crucial role in development and treatment outcome of PD. ${ }^{15,23}$ Hypothetically, each temperament and character dimension is linked to a specific neurotransmitter system and genetic basis. ${ }^{24,25}$ For instance, researches have shown that the dopamine receptor genetic polymorphism connects to NS, ${ }^{26-29}$ whereas serotonin transporter promoter genetic polymorphism relates to HA. ${ }^{30,31}$ In addition, $\mathrm{RD}$ may link to the norepinephrine system. ${ }^{32}$

Researches suggest susceptibility to developing insomnia related to some personality traits, ${ }^{17,18}$ although the idea that all insomniacs have specific personality traits is still controversial. Recently, de Saint Hilaire reported that insomniac patients showed higher HA compared to control subjects. ${ }^{33}$

However, no reports have examined the relationship between personality and insomnia in PD or whether insomniac PD patients show different personality characteristics from those without insomnia. This study aimed to compare temperament and character between insomniacs and non-insomniacs among panic patients.

\section{METHODS}

\section{Subjects}

Participants were 101 patients (58 men and 43 women; aged $41.5 \pm 10.0$ years), recruited from 6 university hospitals in Korea, who met the DSM-IV-TR criteria for PD as determined by structured clinical interview for DSM-IV-TR (SCID). ${ }^{34}$ We excluded patients who satisfied the DSM-IV-TR criteria for major depression or had a lifetime history of schizophrenia, bipolar disorder, posttraumatic stress disorder, obsessive-compulsive disorder, or eating disorder; a history of alcohol or drug abuse or dependence; or a major medical illness. All patients took no medication for at least 2 weeks before participating in the study (or 5 weeks, in the case of fluoxetine). After receiving a complete description of the study, all participants gave written informed consent. This study was approved by the Institutional Review Board of the Samsung Medical Center.

\section{Measures}

We used the Cloninger's Temperament and Character Inventory-Revised-Short (TCI-RS) ${ }^{35}$ to assess personality characteristics and also administered the Panic Disorder Severity Scale (PDSS), ${ }^{36}$ the Hamilton Rating Scale for Anxiety (HAMA), ${ }^{37}$ and 17-item Hamilton Depression Rating Scale (HAMD$17)^{38}$ to all participants. For this study, we defined insomniac patients as those who had score of more than zero on the HAMD-17 sleep item 4 (sleep onset latency), 5 (middle awakening), or 6 (early awakening), whereas non-insomniac patients were defined as those who had only zero score on all three sleep items. We classified insomniacs as three groups; initial, middle and terminal insomnia groups. Regardless of the group overlapping, PD patients who had score of more than zero on item 4,5 and 6 were defined as initial, middle and terminal insomniacs, respectively.

\section{Statistical analyses}

We used the Student's t-test to determine differences between insomniac and non-insomniac panic patients in demographic and clinical characteristics and employed analysis of variance $^{39}$ to examine the relationship between personality and insomnia, controlling for age, sex, and severity of depression (it was defined as total HAMD-17 score minus the sum score of HAMD-17 item 4, 5, and 6) as covariates. The differences in insomnia profiles and personality characteristics between PD with and without agoraphobia were also examined using the Student's t-test. To perform all statistical tests, we used the Statistical Package for the Social Sciences (SPSS) 17.0 (SPSS Inc., Chicago, IL, USA) and we accepted statistical significance for p-values of $<0.017(0.05 / 3)$, by Bonferroni correction.

\section{RESULTS}

\section{Sample characteristics}

Table 1 shows the participants characteristics. Of the 101 panic patients, 20 (19.8\%) panic patients had no insomnia in terms of the HAMD-17 sleep items whereas 81 (80.2\%) panic patients had insomnia. We compared characteristics of panic patients with and without insomnia. There were no significant differences in age, sex, HAM-A score, HAM-D score (total HAMD-17 score minus sum score of item 4-6 to assess the severity of depression) and PDSS score between PD patients with and without insomnia (all p-values $>0.1$ ) (Table 1). Among 101 panic patients, eighty-one patients had any sorts of 
insomnia. Fifty nine patients $(58.4 \%)$ showed initial insomnia, whereas 62 (61.4\%) and 55 (54.5\%) patients showed middle and terminal insomnia, respectively. Patients who have both initial and middle insomnia were 43 (42.6\%), patients who have both initial and terminal insomnia were 40 (39.6\%), patients who have both middle and terminal insomnia were 47 (46.5\%), and patients who have all of initial, middle and terminal insomnia were 35 (34.7\%)(Figure 1).

Table 1. Comparison of demographic and psychological data between panic disorder patients with and without insomnia

\begin{tabular}{lcccc}
\hline & $\begin{array}{c}\text { Panic patients } \\
\text { without insomnia } \\
(\mathrm{N}=20)\end{array}$ & $\begin{array}{c}\text { Panic patients } \\
\text { with insomnia } \\
(\mathrm{N}=81)\end{array}$ & t or $\chi^{2}$ & $\mathrm{p}$ \\
\hline Age & $43.5 \pm 9.9$ & $41.4 \pm 10.5$ & -0.40 & 0.41 \\
Sex (M/F) & $26 / 16$ & $32 / 27$ & 0.44 & 0.54 \\
HAM-A & $11.0 \pm 4.3$ & $10.30 \pm 4.4$ & 0.64 & 0.52 \\
HAM-D* & $10.9 \pm 4.3$ & $10.3 \pm 4.6$ & 0.61 & 0.54 \\
PDSS & $15.5 \pm 4.2$ & $16.7 \pm 4.4$ & 1.40 & 0.17 \\
NS & $31.8 \pm 11.1$ & $28.9 \pm 10.7$ & 1.05 & 0.30 \\
HA & $41.8 \pm 12.3$ & $43.0 \pm 11.0$ & -0.39 & 0.70 \\
RD & $41.2 \pm 8.5$ & $40.3 \pm 6.7$ & 0.46 & 0.65 \\
P & $39.6 \pm 10.5$ & $42.1 \pm 13.0$ & -0.90 & 0.37 \\
SD & $43.5 \pm 10.5$ & $45.2 \pm 11.3$ & -0.63 & 0.53 \\
C & $49.1 \pm 11.2$ & $50.1 \pm 11.3$ & -0.35 & 0.73 \\
ST & $23.9 \pm 11.6$ & $21.0 \pm 12.5$ & 0.99 & 0.32 \\
\hline
\end{tabular}

*total sum of HAMD-17 scores excluding sleep items (4, 5 and 6). HAM-A: Hamilton Rating Scale for Anxiety, HAM-D: 17-item Hamilton Depression Rating Scale, PDSS: Panic Disorder Severity Scale, NS: novelty seeking, HA: harm avoidance, RD: reward dependence, P: persistence, SD: self-directedness, C: cooperativeness, ST: self-transcendence

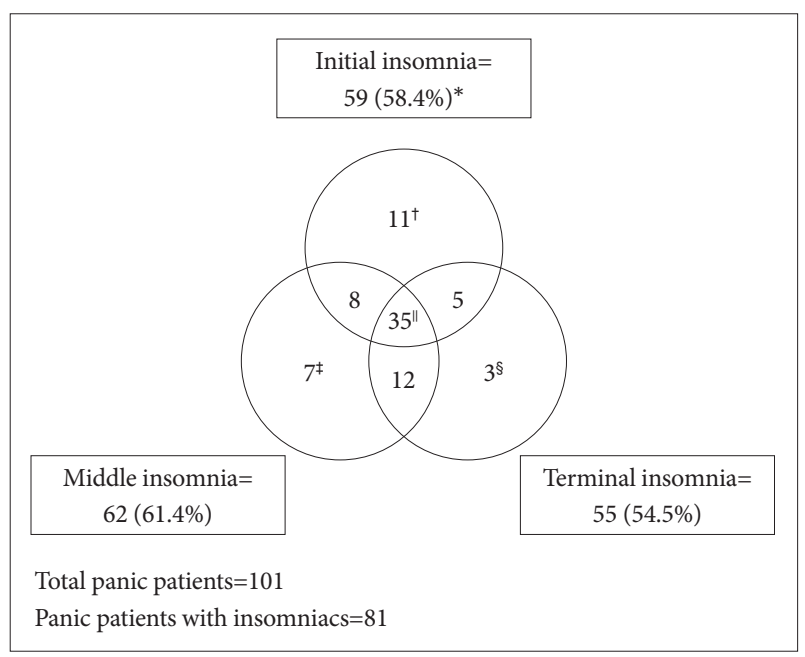

Figure 1. Classification and the number of each insomnia group. *the percentage of each group insomniacs to total panic patients. tinitial insomnia only, ‡middle insomnia only, sterminal insomnia only, "lall stages of initial, middle and terminal insomnia.

\section{Agoraphobia}

There were no significant differences in the TCI variables and prevalence of initial, middle and terminal insomnia between $\mathrm{PD}$ patients with and without agoraphobia (all p-values $>0.1$ ).

\section{Insomnia and personality}

Initial insomnia (delayed sleep onset) correlated significantly to high scores in the temperamental dimension of NS3 $\left(\mathrm{F}_{1,96}=\right.$ 6.93, $\mathrm{p}=0.03) \mathrm{NS} 1, \mathrm{NS} 2$ and NS4 did not show any significance in relation with initial insomnia. ANOVA revealed that low SD score related to initial insomnia $\left(\mathrm{F}_{1,99}=4.2, \mathrm{p}=0.04\right)$, but the significance disappeared after controlling age, sex, HAMA score, severity of depression and PDSS score $(\mathrm{p}=0.1)$. Figure 2 shows the relation between initial insomnia and NS3.

There were no significant ( $\mathrm{p}>0.1$ ) differences in other temperament and character dimensions including NS, HA, RD and $\mathrm{P}$ and SD, C, ST, in case of middle and terminal insomnia with controlling age, sex, HAM-A score, severity of depression and PDSS score.

\section{DISCUSSION}

In the present study, we found that NS3 is related to the development of initial insomnia in panic patients. To our knowledge, this is the first study to compare personality characteristics between panic patients with and without insomnia.

Up to date, many studies have suggested specific personality traits are implicated in insomnia. ${ }^{17,18}$ In addition to, several studies showed that personality disorders were also common in $\mathrm{PD}^{19-21}$ and comorbid personality disorders in PD affect the course and outcome of PD. ${ }^{22,40}$ However, the prevalence and the effect on insomnia of personality disorders in $\mathrm{PD}$ patients are not well known.

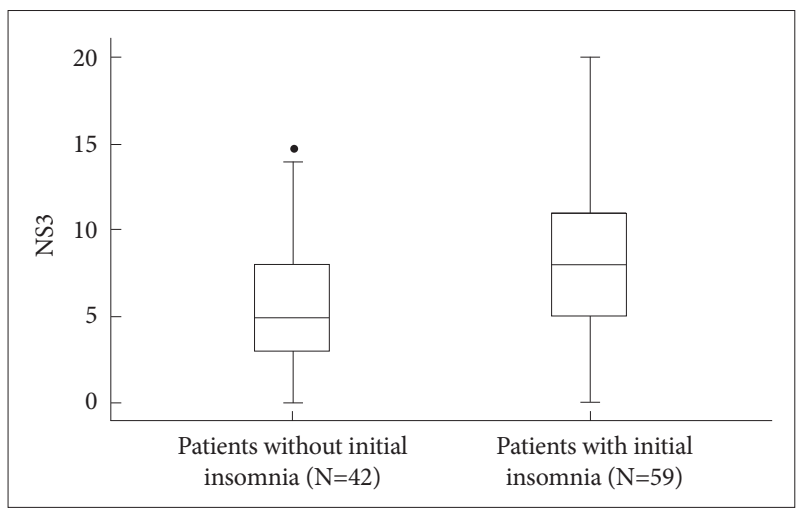

Figure 2. Comparison of novelty-seeking 3 between panic disorder patients with and without initial insomnia $\left(F_{1,96}=6.93\right.$, Bonferroni corrected $p=0.03$ using analysis of variance controlling for age, sex, HAM-A, HAM-D* and PDSS score). *total sum of HAMD-17 scores excluding sleep items $(4,5$, and 6$)$. NS 3 : novelty seeking 3 , HAMA: Hamilton Rating Scale for Anxiety, HAM-D: Hamilton Depression Rating Scale, PDSS: Panic Disorder Severity Scale. 
Cloninger's personality theory ${ }^{14}$ provides an integrated concept for explaining psychopathology of PD, as well as that of insomnia. Many insomniacs have signs of neuroticism, internalization, anxious concerns, and personality traits associated with perfectionism ${ }^{3,41}$ and those characteristics are closely related to HA in the Cloninger's psychobiosocial model..$^{35} \mathrm{HA}$ is characterized by excessive worrying, pessimism, shyness, being fearful and doubtful, and being easily fatigued. Also, biological studies have shown that some genetic polymorphisms in the serotonergic system correlate to HA, as well as to insomnia. ${ }^{42,43}$ Thus, we initially hypothesized that HA would also play an important role in the development of insomnia in the panic patients. However, unexpectedly, our results show that HA is not related to the development of insomnia in PD patients, whereas high NS3 is related to initial insomnia in PD patients.

NS, which has been suggested to be linked to the dopamine system, ${ }^{27,29}$ reflects the tendency towards exploratory activities, lack of inhibition, and impulsiveness. ${ }^{44}$ Thus, individuals with high NS tend to be quick-tempered and more excitable, exploratory, curious, enthusiastic, ardent, easily bored, impulsive, and disorderly. ${ }^{44}$ Although it is unclear why high NS3 is linked to initial insomnia in PD, some hypotheses might possibly explain this finding. Researchers know that the dopaminergic system is connected to the sleep-wake cycle. Wang et al. ${ }^{45}$ reported a correlation between impulsivity scores and mismatch negativity (MMN) in insomniacs, ${ }^{45}$ which suggests a link between the impulsive trait and the arousal mechanism in primary insomnia. Thus, high NS may play a role in the development of initial insomnia in $\mathrm{PD}$ via aberrant dopaminergic function. In addition, people with high NS may have unhealthy, irregular lifestyles, ${ }^{46,47}$ which could act as a mediating factor in the development of insomnia in panic patients. Lifestyle is well-known for playing an important role in the development of both PD and insomnia. Further studies to examine this hypothesis will be needed. ${ }^{48-50}$

Agoraphobia is known to be one of the important prognostic factors in PD. In this study, agoraphobia does not affect either insomnia or personality in PD patients, and we suggest that the relationship between insomnia and personality in $\mathrm{PD}$ is a unique phenomenon regardless of agoraphobia.

As similar with previous researches, our study showed the prevalence rate of $80.2 \%$ of insomnia in PD patients. The prevalence of insomnia in PD patients has been reported about sixty-eight percent up to ninety-three percent, ${ }^{6}$ possibly because of anxiety, depression ${ }^{11}$ and nocturnal panic attack. ${ }^{6} \mathrm{St}-$ ein et al. ${ }^{7}$ reported high prevalence rate of insomnia in patients with PD. At that study, sixty-eight percent among PD patients was reported to have moderate or severe sleep problem compared to only fifteen percent of healthy control. Overbeek et al..$^{51}$ also reported higher prevalence of sleep complaints in PD patients than normal population. There had been a few reports about the difference in the prevalence of insomnia between PD with agoraphobia and PD without agoraphobia. ${ }^{12,13}$ However, any reports did not specify the prevalence of insomnia in PD patients with agoraphobia.

This study had several limitations. First, the sample size was relatively small for generalizing the results to PD. Second, we did not have any healthy controls in this study. Third, we did not measure some important psychosocial variables affecting the course of PD, such as socio-economic status, employment, education, and the consumption of alcoholic beverages and caffeine. Lastly, we did not utilize any specific sleep measures other than sleep items on HAMD-17 and thus might not accurately assess the PD patients' sleep problems. We should have used other sleep questionnaires and polysomnography to specify sleep problems including sleep structure and stages. However, this study is the first attempt to examine the relationship between sleep problems and personality characteristics in PD. Further studies correcting these limitations are needed.

The present study suggests that higher score of NS3 is related to the development of initial insomnia in PD. The clinicians should consider temperament and character when assessing sleep problems in PD.

\section{REFERENCES}

1. Kessler RC, Chiu WT, Jin R, Ruscio AM, Shear K, Walters EE. The epidemiology of panic attacks, panic disorder, and agoraphobia in the National Comorbidity Survey Replication. Arch Gen Psychiatry 2006; 63:415-424.

2. Albert CM, Chae CU, Rexrode KM, Manson JE, Kawachi I. Phobic anxiety and risk of coronary heart disease and sudden cardiac death among women. Circulation 2005;111:480-487.

3. Uhde TW, Cortese BM, Vedeniapin A. Anxiety and sleep problems: emerging concepts and theoretical treatment implications. Curr Psychiatry Rep 2009;11:269-276.

4. Lepola U, Koponen H, Leinonen E. Sleep in panic disorders. J Psychosom Res 1994;38 Suppl 1:105-111.

5. Arriaga F, Paiva T, Matos-Pires A, Cavaglia F, Lara E, Bastos L. The sleep of non-depressed patients with panic disorder: a comparison with normal controls. Acta Psychiatr Scand 1996;93:191-194.

6. Singareddy R, Uhde TW. Nocturnal sleep panic and depression: relationship to subjective sleep in panic disorder. J Affect Disord 2009;112: 262-266.

7. Stein MB, Chartier M, Walker JR. Sleep in nondepressed patients with panic disorder: I. Systematic assessment of subjective sleep quality and sleep disturbance. Sleep 1993;16:724-726.

8. Lydiard RB, Zealberg J, Laraia MT, Fossey M, Prockow V, Gross J, et al. Electroencephalography during sleep of patients with panic disorder. J Neuropsychiatry Clin Neurosci 1989;1:372-376.

9. Mellman TA, Uhde TW. Patients with frequent sleep panic: clinical findings and response to medication treatment. J Clin Psychiatry 1990; 51:513-516.

10. Mellman TA. Sleep and anxiety disorders. Psychiatr Clin North Am 2006;29:1047-1058; abstract x.

11. Lauer CJ, Krieg JC, Garcia-Borreguero D, Ozdaglar A, Holsboer F. Panic disorder and major depression: a comparative electroencephalo- 
graphic sleep study. Psychiatry Res 1992;44:41-54.

12. Lesser IM, Rubin RT, Rifkin A, Swinson RP, Ballenger JC, Burrows GD, et al. Secondary depression in panic disorder and agoraphobia. II. Dimensions of depressive symptomatology and their response to treatment. J Affect Disord 1989;16:49-58.

13. Cordás TA, Ramos RT, Navarro JM, Lotufo Neto F, Gentil V. [Electroencephalographic study of patients with panic disorders]. AMB Rev Assoc Med Bras 1989;35:67-69.

14. Schmidt RE, Gay P, Van der Linden M. Facets of impulsivity are differentially linked to insomnia: evidence from an exploratory study. Behav Sleep Med 2008;6:178-192.

15. Wachleski C, Salum GA, Blaya C, Kipper L, Paludo A, Salgado AP, et al. Harm avoidance and self-directedness as essential features of panic disorder patients. Compr Psychiatry 2008;49:476-481.

16. Starcevic V, Uhlenhuth EH, Fallon S, Pathak D. Personality dimensions in panic disorder and generalized anxiety disorder. J Affect Disord 1996;37:75-79.

17. van de Laar M, Verbeek I, Pevernagie D, Aldenkamp A, Overeem S. The role of personality traits in insomnia. Sleep Med Rev 2010;14:61-68.

18. Lundh LG, Broman JE, Hetta J. Personality traits in patients with persistent insomnia. Pers Individ Dif 1995;18:393-403.

19. Milrod BL, Leon AC, Barber JP, Markowitz JC, Graf E. Do comorbid personality disorders moderate panic-focused psychotherapy? An exploratory examination of the American Psychiatric Association practice guideline. J Clin Psychiatry 2007;68:885-891.

20. Albert U, Maina G, Bergesio C, Bogetto F. Axis I and II comorbidities in subjects with and without nocturnal panic. Depress Anxiety 2006;23: 422-428.

21. Iketani T, Kiriike N, Stein MB, Nagao K, Minamikawa N, Shidao A, et al. Patterns of axis II comorbidity in early-onset versus late-onset panic disorder in Japan. Compr Psychiatry 2004;45:114-120.

22. Ozkan M, Altindag A. Comorbid personality disorders in subjects with panic disorder: do personality disorders increase clinical severity? Compr Psychiatry 2005;46:20-26.

23. Wiborg IM, Falkum E, Dahl AA, Gullberg C. Is harm avoidance an essential feature of patients with panic disorder? Compr Psychiatry 2005; 46:311-314

24. Ando J, Suzuki A, Yamagata S, Kijima N, Maekawa H, Ono Y, et al. Genetic and environmental structure of Cloninger's temperament and character dimensions. J Pers Disord 2004;18:379-393.

25. Samochowiec J, Rybakowski F, Czerski P, Zakrzewska M, Stepień G, Pełka-Wysiecka J, et al. Polymorphisms in the dopamine, serotonin, and norepinephrine transporter genes and their relationship to temperamental dimensions measured by the Temperament and Character Inventory in healthy volunteers. Neuropsychobiology 2001;43:248-253.

26. Ronai Z, Szekely A, Nemoda Z, Lakatos K, Gervai J, Staub M, et al. Association between Novelty Seeking and the $-521 \mathrm{C} / \mathrm{T}$ polymorphism in the promoter region of the DRD4 gene. Mol Psychiatry 2001;6:35-38.

27. Stuettgen MC, Hennig J, Reuter M, Netter P. Novelty Seeking but not BAS is associated with high dopamine as indicated by a neurotransmitter challenge test using mazindol as a challenge substance. Pers Individ Dif 2005;38:1597-1608.

28. Okuyama $Y$, Ishiguro $H$, Nankai M, Shibuya $H$, Watanabe A, Arinami T. Identification of a polymorphism in the promoter region of DRD4 associated with the human novelty seeking personality trait. Mol Psychiatry 2000;5:64-69.

29. Keltikangas-Järvinen L, Elovainio M, Kivimäki M, Lichtermann D, Ekelund J, Peltonen L. Association between the type 4 dopamine receptor gene polymorphism and novelty seeking. Psychosom Med 2003;65: 471-476.
30. Park JW, Kim JS, Lee HK, Kim YI, Lee KS. Serotonin transporter polymorphism and harm avoidance personality in chronic tension-type headache. Headache 2004;44:1005-1009.

31. Ricketts MH, Hamer RM, Sage JI, Manowitz P, Feng F, Menza MA. Association of a serotonin transporter gene promoter polymorphism with harm avoidance behaviour in an elderly population. Psychiatr Genet 1998;8:41-44.

32. Ham BJ, Choi MJ, Lee HJ, Kang RH, Lee MS. Reward dependence is related to norepinephrine transporter T-182C gene polymorphism in a Korean population. Psychiatr Genet 2005;15:145-147.

33. de Saint Hilaire Z, Straub J, Pelissolo A. Temperament and character in primary insomnia. Eur Psychiatry 2005;20:188-192.

34. First MB, Spitzer RL, Gibbon M, Williams JBW. Structured Clinical Interview for DSM-IV Axis I Disorders. New York: New York State Psychiatric Institute; 1996.

35. Cloninger CR. Temperament and personality. Curr Opin Neurobiol 1994;4:266-273.

36. Lim YJ, Yu BH, Kim JH. Korean panic disorder severity scale: construct validity by confirmatory factor analysis. Depress Anxiety 2007; 24:95-102.

37. Hamilton M. The assessment of anxiety states by rating. Br J Med Psychol 1959;32:50-55.

38. Hamilton M. A rating scale for depression. J Neurol Neurosurg Psychiatry 1960;23:56-62.

39. Litvitskiĭ PF, Kogan AKh, Kudrin AN, Luk'ianova LO, Ol’binskaia LI. [Suppression of myocardial lipid peroxidation during preventive treatment of transitory coronary insufficiency with myophedrine]. Farmakol Toksikol 1981;44:303-307.

40. Friedman S, Jones JC, Chernen L, Barlow DH. Suicidal ideation and suicide attempts among patients with panic disorder: a survey of two outpatient clinics. Am J Psychiatry 1992;149:680-685.

41. van de Laar M, Verbeek I, Pevernagie D, Aldenkamp A, Overeem S. The role of personality traits in insomnia. Sleep Med Rev 2010;14:61-68.

42. Perlis RH, Mischoulon D, Smoller JW, Wan YJ, Lamon-Fava S, Lin KM, et al. Serotonin transporter polymorphisms and adverse effects with fluoxetine treatment. Biol Psychiatry 2003;54:879-883.

43. Brummett BH, Krystal AD, Ashley-Koch A, Kuhn CM, Züchner S, Siegler IC, et al. Sleep quality varies as a function of 5-HTTLPR genotype and stress. Psychosom Med 2007;69:621-624.

44. Cloninger CR, Svrakic DM, Przybeck TR. A psychobiological model of temperament and character. Arch Gen Psychiatry 1993;50:975-990.

45. Wang W, Zhu SZ, Pan LC, Hu AH, Wang YH. Mismatch negativity and personality traits in chronic primary insomniacs. Funct Neurol 2001; 16:3-10.

46. Fergusson DM, Boden JM, Horwood LJ. The developmental antecedents of illicit drug use: evidence from a 25-year longitudinal study. Drug Alcohol Depend 2008;96:165-177.

47. Sovio U, King V, Miettunen J, Ek E, Laitinen J, Joukamaa M, et al. Cloninger's Temperament dimensions, socio-economic and lifestyle factors and metabolic syndrome markers at age 31 years in the Northern Finland Birth Cohort 1966. J Health Psychol 2007;12:371-382.

48. Goodwin RD, Keyes KM, Stein MB, Talley NJ. Peptic ulcer and mental disorders among adults in the community: the role of nicotine and alcohol use disorders. Psychosom Med 2009;71:463-468.

49. Bixler E. Sleep and society: an epidemiological perspective. Sleep Med 2009;10 Suppl 1:S3-S6.

50. Neubauer DN. Current and new thinking in the management of comorbid insomnia. Am J Manag Care 2009;15 Suppl:S24-S32.

51. Overbeek T, van Diest R, Schruers K, Kruizinga F, Griez E. Sleep complaints in panic disorder patients. J Nerv Ment Dis 2005;193:488-493. 